\title{
Anti-malarial Activity of New Emodin Derivatives against Plasmodium falciparum Chloroquine Resistant Strain \\ Sukanya Pandeti ${ }^{1}$, Sarika Gunjan ${ }^{2}$, Sandeep Paidipelli ${ }^{1}$, Renu Tripathi ${ }^{2}$ and Narender Tadigoppula ${ }^{1 *}$
}

${ }^{1}$ Medicinal and Process Chemistry Division, CSIR-Central Drug Research Institute, Lucknow-226 031, U.P India

${ }^{2}$ Parasitology Division, CSIR-Central Drug Research Institute, Lucknow-226 031, U.P, India

\begin{abstract}
Emodin (1) is the major bioactive compound of several herb species, which belongs to anthraquinone class of compound. As a part of our drug discovery program, large quantities of emodin (1) was isolated from the roots of Rheum emodi and a library of novel emodin derivatives 2-24 were prepared to evaluate their in-vitro antimalarial activity, among them, compound 17, 18 and 20 showed potent antimalarial activity against chloroquine resistant strain PfK1with the IC50 of $2.28,2.49$ and $2.48 \mu \mathrm{M}$ respectively with a high safety index.
\end{abstract}

\section{Keywords: Emodin derivatives; Antimalarial agents;} Anthraquinones; Cytotoxicity; Rheum emodi

\section{Introduction}

Anthraquinones are widely distributed secondary metabolites of plants (aloe, cascara sagrada, senna and rhubarb), microbes, lichens and insects, which possess various biological activities [1-3]. Emodin (1) an anthraquinone class of compound is the major bioactive compounds of numerous herb species such as Rheum, Polygonum (polygonaceae), Rhamnus (Rhamnaceae) and Cassieae (senna) [4-6] and possesses immunosuppressive, anticancer [7], anti-inflammatory, anti-atherosclerotic, and vasorelaxant effects [8-11].

As a part of our drug discovery program on antimalarial agents from Indian medicinal plants, we isolated large quantities of anthraquinone i.e. emodin (1) from the roots of Rheum emodi and planned to carry out chemical transformation to improve its therapeutic application. Chemical transformation of bioactive compounds of medicinal herbs is one of the most common approaches in drug discovery to improve the therapeutic properties. Towards this goal, we have synthesized a library of novel emodin derivatives 2-24 and their antimalarial activity was evaluated.

\section{Materials and Methods}

\section{General chemistry}

IR spectra were recorded on perkin-Elmer RX-1 spectrometer. Using either $\mathrm{KBr}$ pellets (or) in neat. ${ }^{1} \mathrm{H}-\mathrm{NMR},{ }^{13} \mathrm{C}-\mathrm{NMR}$, DEPT-90 and DEPT-135 spectra were run on Bruker Advance DPX $300 \mathrm{MHz}$ and $200 \mathrm{MHz}$ in $\mathrm{CDCl}_{3}$. Chemical shifts are reported as values in ppm relative to $\mathrm{CHCl}_{3}(7.26)$ in $\mathrm{CDCl}_{3}$ and TMS was used as internal standard.ESI mass spectra were recorded on JEOL SX 102/DA-6000. Chromatography was executed with silica gel (60-120 mesh) using mixtures of chloroform, ethyl acetate and hexane as eluants.

\section{Background of plant}

Rheum emodi wall (Family: Polygonaceae, commonly known as revand-chini and English name rhubarb) is a stout herb, distributed in the alpine and sub-alpine zones of the Himalayas. The roots of this species are used widely in ayurvedic medicine. Roots of the Indian rhubarb is darker, inferior in aroma, and is a well-known stomachic, bitter, cathartic and used all over the world.

\section{Collection of medicinal plant}

Rheum emodi wall (Bark) was purchased from the local market of Lucknow, U.P, India and the authentification was done by Botany Division of Central Drug Research Institute, Lucknow.

\section{Extraction}

Powdered Rheum emodi wall (Bark) (3 kg) were placed in glass percolator with $95 \%$ ethanol (10 lit) and allowed to stand for $24 \mathrm{hr}$ at room temperature. The percolate was collected and these processes were repeated for four times. The combined percolate was evaporated under reduced pressure at $50^{\circ} \mathrm{C}$ to afford ethanol extract. The weight of extract was found to be $200 \mathrm{~g}$.

\section{Isolation and purification of Emodin}

The alcoholic extract was (200 g) chromatographed on a column of silica gel (60-120 mesh), eluted with hexane and chloroform (70:30); recrystallization from methanol afford emodin (3g). The compound visualization was obtained under UV light, also shown orange spot by spraying with $10 \%$ sulphuric acid in methanol.

\section{1, 3, 8-trihydroxy-6-methylanthracene-9-10-dione (1)}

IR (KBr) 3613, 2925, 1625, 1461, 1373, 1277, 1029, 767, $672 \mathrm{~cm}$ '; ${ }^{1} \mathrm{H}$ NMR (DMSO-d, $\left.300 \mathrm{MHz}\right) \delta 12.03(\mathrm{~s}, \mathrm{OH}), 11.95(\mathrm{~s}, \mathrm{OH})$, $7.41(\mathrm{~s}, 1 \mathrm{H}), 7.10(\mathrm{~s}, 1 \mathrm{H}), 7.06(\mathrm{~s}, 1 \mathrm{H}), 6.55(\mathrm{~s}, 1 \mathrm{H}), 2.38(\mathrm{~s}, 3 \mathrm{H}) ;{ }^{13} \mathrm{C}$ NMR(DMSO-d, $75 \mathrm{MHz}) \delta 189.40,180.92,165.51,164.35,161.30$, $148.04,134.77,132.47,123.91,120.25,113.04,108.09,107.75,21.45$ MS (ESI) m/z 270.

\section{Preparation of emodin derivatives}

General method for O-alkylation (Method A): A stirred solution of compound $\mathbf{1}$ (100 mg, 0.00037 moles) in DMF (5 mL) at room temperature was treated with respective alkyl halide (RX) $(0.00033$ moles) and $\mathrm{K}_{2} \mathrm{CO}_{3}(0.00092$ moles $)$. The reaction mixture was stirred at $60-70^{\circ} \mathrm{C}$ for $4 \mathrm{hr}$. It was then extracted with ethyl acetate $(3 \times 25 \mathrm{~mL})$, the organic layer was washed with water, dried over anhydrous $\mathrm{Na}_{2} \mathrm{SO}_{4}$

*Corresponding author: Narender Tadigoppula, Medicinal and Process Chemistry Division, CSIR - Central Drug Research Institute, Lucknow-226031, U.P, India, Tel: +91-5222612411; Fax: +91-5222623405; E-mail: t_narendra@cdri.res.in

Received September 18, 2014; Accepted September 26, 2014; Published September 28, 2014

Citation: Pandeti S, Gunjan S, Paidipelli S, Tripathi R, Tadigoppula N (2014) Anti-malarial Activity of New Emodin Derivatives against Plasmodium falciparum Chloroquine Resistant Strain. Nat Prod Chem Res 2: 150. doi:10.4172/2329-6836.1000150

Copyright: ( $) 2014$ Pandeti S, et al.. This is an open-access article distributed under the terms of the Creative Commons Attribution License, which permits unrestricted use, distribution, and reproduction in any medium, provided the original author and source are credited. 
Citation: Pandeti S, Gunjan S, Paidipelli S, Tripathi R, Tadigoppula N (2014) Anti-malarial Activity of New Emodin Derivatives against Plasmodium falciparum Chloroquine Resistant Strain. Nat Prod Chem Res 2: 150. doi:10.4172/2329-6836.1000150

and evaporated under reduced pressure. Then the crude product was chromatographed on silica gel to afford the desired compound.

Ethyl 2 - (4, 5-dihydroxy-7-methyl-9, 10-dioxo-9, 10 dihydroanthracen-2-yloxy)-2-methylpropanoate (2): IR ( $\mathrm{KBr}) 3688,3400$, $2374,1722,1625,1468,1382,1283,1217,1133,766,671 \mathrm{~cm}^{-1} ;{ }^{1} \mathrm{H}$ NMR $\left(\mathrm{CDCl}_{3} 300 \mathrm{MHz}\right) \delta 12.22(\mathrm{~s}, \mathrm{OH}), 12.10(\mathrm{~s}, \mathrm{OH}), 8.02(\mathrm{~s}, 1 \mathrm{H}), 7.62(\mathrm{~s}$, $1 \mathrm{H}), 7.09(\mathrm{~s}, 1 \mathrm{H}), 6.56(\mathrm{~s}, 1 \mathrm{H}), 4.31-4.24(\mathrm{q}, 2 \mathrm{H}), 2.45(\mathrm{~s}, 3 \mathrm{H}), 1.71(\mathrm{~s}$ $6 \mathrm{H}), 1.42(\mathrm{t}, 3 \mathrm{H})$; ESI - MS: Cacld for $\mathrm{C}_{21} \mathrm{H}_{20} \mathrm{O}_{7}[\mathrm{M}+\mathrm{H}]^{+}: 384$, Found 384; Yield: $65 \%$.

3-(2-(diethyl amino) ethoxy)-1, 8-dihydroxy-6-methylanthracene-9, 10-dione (3): IR (KBr) 3869, 3413, 1638,1333, 1216, 1028, 925, $764,672 \mathrm{~cm}^{-1} ;{ }^{1} \mathrm{H}$ NMR $\left(\mathrm{CDCl}_{3}, 300 \mathrm{MHz},\right) \delta 12.27(\mathrm{~s}, \mathrm{OH}), 12.09(\mathrm{~s}$, $\mathrm{OH}), 7.61(\mathrm{~s}, 1 \mathrm{H}), 7.35(\mathrm{~s}, 1 \mathrm{H}), 7.07(\mathrm{~s}, 1 \mathrm{H}), 6.68(\mathrm{~s}, 1 \mathrm{H}), 4.25(\mathrm{t}, 2 \mathrm{H})$, $2.86(\mathrm{t}, 2 \mathrm{H}), 2.62(\mathrm{~s}, 4 \mathrm{H}), 2.45(\mathrm{~s}, 3 \mathrm{H}), 1.40(\mathrm{t}, 6 \mathrm{H})$; ESI - MS: Cacld for $\mathrm{C}_{21} \mathrm{H}_{23} \mathrm{NO}_{5}[\mathrm{M}+\mathrm{H}]^{+}: 370$, Found 370; Yield: $70 \%$.

1,8-dihydroxy-3-methyl-6-(2-(pyrrolidin-1-yl)ethoxy) anthracene-9, 10-dione (4): IR (KBr) 3438, 2341, 1720, 1630, 1463, $1385,1285,1218,1130,1074,765,668 \mathrm{~cm}^{-1} ;{ }^{1} \mathrm{H} \mathrm{NMR}\left(\mathrm{CDCl}_{3}, 300 \mathrm{MHz}\right.$, $\delta 7.59(\mathrm{~s}, 1 \mathrm{H}), 7.32(\mathrm{~s}, 1 \mathrm{H}), 7.05(\mathrm{~s}, 1 \mathrm{H}), 6.66(\mathrm{~s}, 1 \mathrm{H}), 4.29(\mathrm{t}, 2 \mathrm{H}), 2.95$ $(\mathrm{t}, 2 \mathrm{H}), 2.66(\mathrm{t}, 4 \mathrm{H}), 2.43(\mathrm{~s}, 3 \mathrm{H}), 2.04(\mathrm{t}, 4 \mathrm{H})$; ESI - MS: Cacld for $\mathrm{C}_{21} \mathrm{H}_{21} \mathrm{NO}_{5}[\mathrm{M}+\mathrm{H}]^{+}: 368$, Found 368; Yield: 68\%.

1, 8-dihydroxy-3-methyl-6-(2-(piperidin-1-yl) ethoxy) anthracene-9, 10-dione (5): IR ( $\mathrm{KBr})$ 3285, 2923, 1725, 1623, 1461, 1376, 1218, 909, 764, $669 \mathrm{~cm}^{-1} ;{ }^{1} \mathrm{H} \mathrm{NMR}\left(\mathrm{CDCl}_{3}, 300 \mathrm{MHz}\right.$, $\delta 7.61(\mathrm{~s}, 1 \mathrm{H})$, $7.35(\mathrm{~s}, 1 \mathrm{H}), 7.08(\mathrm{~s}, 1 \mathrm{H}), 6.68(\mathrm{~s}, 1 \mathrm{H}), 4.31(\mathrm{t}, 2 \mathrm{H}), 2.96(\mathrm{t}, 2 \mathrm{H}), 2.68(\mathrm{t}$, $4 \mathrm{H}), 2.45(\mathrm{~s}, 3 \mathrm{H}), 1.43(\mathrm{t}, 6 \mathrm{H})$; ESI - MS: Cacld for $\mathrm{C}_{22} \mathrm{H}_{23} \mathrm{NO}_{5}[\mathrm{M}+\mathrm{H}]^{+}$: 383, Found 383; Yield: 68\%.

1, 8-dihydroxy-3-methyl-6-(2-morpholinoethoxy) anthracene-9, 10-dione (6): IR (KBr) 3677, 3646, 3018, 1626, 1462, 1217, 920, 767, $670 \mathrm{~cm}^{-1} ;{ }^{1} \mathrm{H}$ NMR $\left(\mathrm{CDCl}_{3}, 300 \mathrm{MHz},\right) \delta 7.60(\mathrm{~s}, 1 \mathrm{H}), 7.34(\mathrm{~s}, 1 \mathrm{H}), 7.06$ $(\mathrm{s}, 1 \mathrm{H}), 6.67(\mathrm{~s}, 1 \mathrm{H}), 4.24(\mathrm{t}, 2 \mathrm{H}), 3.74(\mathrm{t}, 4 \mathrm{H}), 2.85(\mathrm{t}, 3 \mathrm{H}), 2.62(\mathrm{t}, 4 \mathrm{H})$, $2.44(\mathrm{~s}, 3 \mathrm{H})$; ESI - MS: Cacld for $\mathrm{C}_{21} \mathrm{H}_{21} \mathrm{NO} 6[\mathrm{M}+\mathrm{H}]^{+}: 384$, Found 384; Yield: 70\%.

1, 8-dihydroxy-3-methyl-6-(nonyloxy) anthracene-9, 10-dione (7): IR (KBr) 3365, 2359, 1628, 1461, 1217, 1020, 769, $671 \mathrm{~cm}^{-1} ;{ }^{1} \mathrm{H}$ NMR $\left(\mathrm{CDCl}_{3} 300 \mathrm{MHz}\right) \delta 12.27(\mathrm{~s}, \mathrm{OH}), 12.10(\mathrm{~s}, \mathrm{OH}), 7.59(\mathrm{~s}, 1 \mathrm{H})$, $7.32(\mathrm{~s}, 1 \mathrm{H}), 7.05(\mathrm{~s}, 1 \mathrm{H}), 6.64(\mathrm{~s}, 1 \mathrm{H}), 4.07(\mathrm{t}, 2 \mathrm{H}), 2.43(\mathrm{~s}, 3 \mathrm{H}), 1.82$ $1.78(\mathrm{~m}, 2 \mathrm{H}), 1.58(\mathrm{~s}, 2 \mathrm{H}), 1.45(\mathrm{~s}, 2 \mathrm{H}), 1.25(\mathrm{~s}, 6 \mathrm{H}), 0.85(\mathrm{t}, 3 \mathrm{H}) ; \mathrm{MS}$ (ESI) $\mathrm{m} / \mathrm{z}$ 382; Yield:66\%.

1, 8-dihydroxy-3-methyl-6-(undecyoxy) anthracene-9, 10-dione (8): IR (KBr) 3761, 3414, 2952, 1624, 1318, 1216, 766, $671 \mathrm{~cm}^{-1} ;{ }^{1} \mathrm{H}$ NMR ( $\mathrm{CDCl}_{3}, 300 \mathrm{MHz}$,) $\delta 12.49(\mathrm{~s}, \mathrm{OH}), 12.32(\mathrm{~s}, \mathrm{OH}), 7.81(\mathrm{~s}, 1 \mathrm{H})$, $7.54(\mathrm{~s}, 1 \mathrm{H}), 7.46(\mathrm{~s}, 1 \mathrm{H}), 6.84(\mathrm{~s}, 1 \mathrm{H}), 4.27(\mathrm{t}, 2 \mathrm{H}), 2.64(\mathrm{~s}, 3 \mathrm{H}), 2.02$ - $1.99(\mathrm{~m}, 2 \mathrm{H}), 1.76(\mathrm{~s}, 2 \mathrm{H}), 1.66(\mathrm{~s}, 2 \mathrm{H}), 1.45(\mathrm{~s}, 16 \mathrm{H}), 1.07(\mathrm{t}, 3 \mathrm{H})$ ); ${ }^{13} \mathrm{C}$ NMR( $\left.\mathrm{CDCl}_{3}, 75 \mathrm{MHz}\right) \delta 191.06,183.04,167.18,166.19,163.45$, $149.31,140.27,136.16,134.23,125.44,122.21,115.06,114.70,111.00$, $109.72,108.10,70.04,34.02,32.93,32.63,30.70,30.50,30.53,30.36$, 30.29, 30.16, 29.96, 29.89, 26.89, 23.69, 23.13, 15.11; MS (ESI) m/z 438; Yield: $65 \%$.

3-(dodecyloxy)-1, 8-dihydroxy-6-methyl anthracene-9, 10-dione (9): IR (KBr) 3429, 1627, 1473, 1386, 1303, 1264, 1218, 1032, 769, 670 $\mathrm{cm}^{-1} ;{ }^{1} \mathrm{H}$ NMR $\left(\mathrm{CDCl}_{3}, 300 \mathrm{MHz}\right.$ ) $\delta 12.30(\mathrm{~s}, \mathrm{OH}), 12.13(\mathrm{~s}, \mathrm{OH}), 7.62$ (s, 1H), $7.35(\mathrm{~s}, 1 \mathrm{H}), 7.07(\mathrm{~s}, 1 \mathrm{H}), 6.85(\mathrm{~s}, 1 \mathrm{H}), 4.08(\mathrm{t}, 2 \mathrm{H}), 2.44(\mathrm{~s}, 3 \mathrm{H})$, $2.05(\mathrm{~s}, 2 \mathrm{H}), 1.85-1.80(\mathrm{~m}, 2 \mathrm{H}), 1.57(\mathrm{~s}, 2 \mathrm{H}), 1.45-1.41(\mathrm{~m}, 4 \mathrm{H}), 1.29(\mathrm{~s}$,
2OH), $0.86(\mathrm{t}, 3 \mathrm{H}) ;{ }^{13} \mathrm{C} \mathrm{NMR}\left(\mathrm{CDCl}_{3}, 75 \mathrm{MHz}\right) \delta 190.65,182.01,166.16$, $165.17,162.44,148.31,136.12,133.19,124.43,121.21,106.71,107.08$, $69.03,31.92,31.62,29.66,29.36,28.88,25.88,22.69,22.13,14.12$; MS (ESI) $\mathrm{m} / \mathrm{z}$ 452; Yield: 65\%.

1,8-dihydroxy-3-methyl-6-(pentadecyloxy)anthracene-9, 10-dione (10): IR (KBr) 3490, 2924, 1627, 1452, 1217, 769, $670 \mathrm{~cm}^{-1}$; ${ }^{1} \mathrm{H}$ NMR $\left(\mathrm{CDCl}_{3}, 300 \mathrm{MHz}\right.$, $\delta 7.61(\mathrm{~s}, 1 \mathrm{H}), 7.34(\mathrm{~s}, 1 \mathrm{H}), 7.06(\mathrm{~s}, 1 \mathrm{H})$, $6.65(\mathrm{~s}, 1 \mathrm{H}), 4.07(\mathrm{t}, 2 \mathrm{H}), 2.44(\mathrm{~s}, 3 \mathrm{H}), 2.02(\mathrm{t}, 2 \mathrm{H}), 1.84-1.79(\mathrm{~m}, 2 \mathrm{H})$, $1.56(\mathrm{~s}, 2 \mathrm{H}), 1.25(\mathrm{~s}, 24 \mathrm{H}), 0.87(\mathrm{t}, 3 \mathrm{H}) ;{ }^{13} \mathrm{C} \mathrm{NMR}\left(\mathrm{CDCl}_{3}, 75 \mathrm{MHz}\right) \delta$ $190.69,182.05,166.19,166.20,162.47,148.32,139.27,135.17,133.24$, $124.45,121.22,114.06,113.70,110.03,108.72,107.10,69.04,33.83$, $31.93,31.63,29.70,29.54,29.37,29.30,29.16,28.96,28.89,25.89,22.70$, 22.13, 14.12; MS (ESI) m/z 494; Yield: 62\%.

1,8-dihydroxy-3-methyl-6-(octadecyloxy)anthracene-9, 10-dione (11): IR (KBr) 3407, 2952, 1631, 1363, 1217, 1012, 769, 670 $\mathrm{cm}^{-1} ;{ }^{1} \mathrm{H}$ NMR $\left(\mathrm{CDCl}_{3}, 300 \mathrm{MHz},\right) \delta 12.29(\mathrm{~s}, \mathrm{OH}), 12.12(\mathrm{~s}, \mathrm{OH}), 7.61$ (s, 1H), $7.34(\mathrm{~s}, 1 \mathrm{H}), 7.07(\mathrm{~s}, 1 \mathrm{H}), 6.66(\mathrm{~s}, 1 \mathrm{H}), 4.09(\mathrm{t}, 2 \mathrm{H}), 2.45(\mathrm{~s}, 3 \mathrm{H})$, $1.86-1.82(\mathrm{~m}, 2 \mathrm{H}), 1.60(\mathrm{~s}, 2 \mathrm{H}), 1.47-1.43(\mathrm{~m}, 2 \mathrm{H}), 1.27(\mathrm{~s}, 28 \mathrm{H}), 0.87$ $(\mathrm{t}, 3 \mathrm{H}) ; \mathrm{MS}$ (ESI) $\mathrm{m} / \mathrm{z}$ 522; Yield: $65 \%$.

1, 8-dihydroxy-3-methyl-6-(icosyloxy) anthracene-9, 10-dione (12): IR (KBr) 3289, 2368, 1658, 1584, 1458, 1218, 769, $670 \mathrm{~cm}^{-1} ;{ }_{1} \mathrm{H}$ $\operatorname{NMR}\left(\mathrm{CDCl}_{3}, 300 \mathrm{MHz}\right.$ ) $\delta 12.31(\mathrm{~s}, \mathrm{OH}), 12.15(\mathrm{~s}, \mathrm{OH}), 7.64(\mathrm{~s}, 1 \mathrm{H})$, $7.37(\mathrm{~s}, 1 \mathrm{H}), 7.09(\mathrm{~s}, 1 \mathrm{H}), 6.68(\mathrm{~s}, 1 \mathrm{H}), 4.10(\mathrm{t}, 2 \mathrm{H}), 2.46(\mathrm{~s}, 3 \mathrm{H}), 2.05(\mathrm{t}$, $2 \mathrm{H}), 1.87-1.82(\mathrm{~m}, 2 \mathrm{H}), 1.59(\mathrm{~s}, 2 \mathrm{H}), 1.34(\mathrm{~s}, 31 \mathrm{H}), 0.88(\mathrm{t}, 3 \mathrm{H})) ;{ }^{13} \mathrm{C}$ $\mathrm{NMR}\left(\mathrm{CDCl}_{3}, 75 \mathrm{MHz}\right) \delta 190.11,181.47,165.61,164.62,161.89,147.74$, $138.69,134.59,132.66,123.87,120.54,113.48,113.12,109.45,108.14$, $106.52,68.45,32.25,31.35,31.05,29.12,29.0,28.79,28.72,28.59,28.38$, 28.31, 25.31, 22.12, 21.55, 13.54; MS (ESI) m/z 550; Yield: 63\%.

3-(docosyloxy)-1, 8-dihydroxy-6-methyl anthracene-9, 10-dione (13): $\mathrm{IR}(\mathrm{KBr}) 3388,2362,1624,1365,1217,767,672 \mathrm{~cm}^{-1} ;{ }^{1} \mathrm{H}$ NMR $\left(\mathrm{CDCl}_{3}, 300 \mathrm{MHz}\right.$ ) $\delta 12.29(\mathrm{~s}, \mathrm{OH}), 12.12(\mathrm{~s}, \mathrm{OH}), 7.61(\mathrm{~s}, 1 \mathrm{H}), 7.34$ $(\mathrm{s}, 1 \mathrm{H}), 7.06(\mathrm{~s}, 1 \mathrm{H}), 6.65(\mathrm{~s}, 1 \mathrm{H}), 4.08(\mathrm{t}, 2 \mathrm{H}), 2.44(\mathrm{~s}, 3 \mathrm{H}), 2.05(\mathrm{t}, 2 \mathrm{H})$, $1.82(\mathrm{~s}, 2 \mathrm{H}), 1.25$ (s, 34H), 0.88 (t, 3H); MS (ESI) m/z 578; Yield: 64\%.

(E)-3-(3, 7-dimethylocta-2, 6-dienyloxy) 1, 8-dihydroxy-6methyl anthracene-9, 10-Dione (14): IR (KBr) 3349, 2367, 1718, 1624, 1364, 1218, 770, $650 \mathrm{~cm}^{-1}$; ${ }^{1} \mathrm{H}$ NMR $\left(\mathrm{CDCl}_{3}, 300 \mathrm{MHz},\right) \delta^{1} \mathrm{H} 12.32(\mathrm{~s}$, $\mathrm{OH}), 12.16(\mathrm{~s}, \mathrm{OH}), 7.64(\mathrm{~s}, 1 \mathrm{H}), 7.28(\mathrm{~s}, 1 \mathrm{H}), 7.10(\mathrm{~s}, 1 \mathrm{H}), 6.70(\mathrm{~s}, 1 \mathrm{H})$, $5.51(\mathrm{t}, 1 \mathrm{H}), 5.11(\mathrm{~s}, 1 \mathrm{H}), 4.71(\mathrm{t}, 2 \mathrm{H}), 2.47(\mathrm{~s}, 3 \mathrm{H}), 2.14(\mathrm{~s}, 4 \mathrm{H}), 1.80(\mathrm{~s}$, $6 \mathrm{H}), 1.73$ (s, 3H); MS (ESI) m/z 406; Yield: 62\%.

1, 8-dihydroxy-3-methyl-6-(prop-2-ynyloxy) anthracene-9, 10-dione (15): IR (KBr) 3282, 2922, 2125, 2357, 1717, 1631, 1390, 1218 , $1078,766,680 \mathrm{~cm}^{-1} ;{ }^{1} \mathrm{H}$ NMR $\left(\mathrm{CDCl}_{3}, 300 \mathrm{MHz}\right) \delta 12.12(\mathrm{~s}, \mathrm{OH}), 12.01$ (s, OH), $7.80(\mathrm{~s}, 1 \mathrm{H}), 7.66(\mathrm{~s}, 1 \mathrm{H}), 7.64(\mathrm{~s}, 1 \mathrm{H}), 7.096(\mathrm{~s}, 1 \mathrm{H}), 4.03(\mathrm{~s}$, 2H), 2.85 (s, 1H), 2.46 (s, 3H); MS (ESI) m/z 308; Yield: 60\%.

\section{General method for C-alkylation (Method B)}

A stirred solution of amine $(0.0022$ moles $)$ cooled at $0^{\circ} \mathrm{C}$ was slowly treated with formalin (0.00148 moles), glacial acetic acid $(0.0192$ moles) and compound 1 (100 mg, 0.00037 moles). The whole reaction mixture was brought to room temperature and stirred for $4 \mathrm{hr}$, after $\mathrm{pH}$ was adjusted to 8 by addition of $20 \%$ aq. $\mathrm{NaOH}$. It was then extracted with ethyl acetate $(3 \times 25 \mathrm{~mL})$, the organic layer was washed with water, dried over anhydrous $\mathrm{Na}_{2} \mathrm{SO}_{4}$ and evaporated under reduced pressure. Then the crude product was chromatographed on silica gel to afford the desired compound.

1,3,8-trihydroxy-6-methyl-2-(pyrrolidin-1-ylmethyl) anthracene-9, 10-dione (16): IR (KBr) 3556, 3023, 2925, 2357, 1625, 1379, 1278, 1104, 767, $671 \mathrm{~cm}^{-1} ;{ }^{1} \mathrm{H}$ NMR (DMSO-d $\left.{ }^{6}, 300 \mathrm{MHz}\right) \delta 7.40$ 
(s, 1H), $6.85(\mathrm{~s}, 1 \mathrm{H}), 6.78(\mathrm{~s}, 1 \mathrm{H}), 4.14(\mathrm{~s}, 2 \mathrm{H}), 3.21(\mathrm{t}, 4 \mathrm{H}), 2.36(\mathrm{~s}, 3 \mathrm{H})$ $1.96(\mathrm{t}, 4 \mathrm{H})$; ESI - MS: Cacld for $\mathrm{C}_{20} \mathrm{H}_{19} \mathrm{NO}_{5}[\mathrm{M}+\mathrm{H}]^{+}: 354$, Found 354; Yield: $80 \%$.

1,3,8-trihydroxy-6-methyl-2-(piperidin-1-ylmethyl) anthracene-9, 10-dione (17): $\operatorname{IR}(\mathrm{KBr}) 3401,2927,2372,1628,1535$, 1459, 1369, 1222, 1112, $768 \mathrm{~cm}^{-1},{ }^{1} \mathrm{H}$ NMR (DMSO-d $\left.\mathrm{d}^{6}, 300 \mathrm{MHz}\right) \delta 7.39$ (s, $1 \mathrm{H}), 6.90(\mathrm{~s}, 2 \mathrm{H}), 3.90(\mathrm{~s}, 2 \mathrm{H}), 2.74(\mathrm{t}, 4 \mathrm{H}), 2.33(\mathrm{~s}, 3 \mathrm{H}), 1.68(\mathrm{t}, 4 \mathrm{H})$, $1.52(\mathrm{t}, 2 \mathrm{H})$; ESI - MS: Cacld for $\mathrm{C}_{21} \mathrm{H}_{21} \mathrm{NO}_{5}[\mathrm{M}+\mathrm{H}]^{+}: 369$, Found 369; Yield: $78 \%$.

$1,3,8$ - trihydroxy-6-methyl-2-( morpholinomethyl) anthracene-9, 10-dione (18): IR (KBr) 3565, 3021, 2929, 2357, 1720, $1618,1461,1377,1283,1217,1122,765,671 \mathrm{~cm}^{-1} ;{ }^{1} \mathrm{H}$ NMR $\left(\mathrm{CDCl}_{3} \&\right.$ DMSO-d $\left.^{6}, 300 \mathrm{MHz}\right) \delta 7.43(\mathrm{~s}, 1 \mathrm{H}), 6.86(\mathrm{~s}, 1 \mathrm{H}), 6.70(\mathrm{~s}, 1 \mathrm{H}), 3.65(\mathrm{t}$, $2 \mathrm{H}), 3.49(\mathrm{t}, 4 \mathrm{H}), 2.54(\mathrm{t}, 2 \mathrm{H}), 2.27(\mathrm{t}, 2 \mathrm{H}), 2.17(\mathrm{~s}, 3 \mathrm{H})$; ESI - MS: Cacld for $\mathrm{C}_{21} \mathrm{H}_{23} \mathrm{NO}_{5}[\mathrm{M}+\mathrm{H}]^{+}: 370$, Found 370; ESI - MS: Cacld for $\mathrm{C}_{20} \mathrm{H}_{19} \mathrm{NO}_{6}$ $[\mathrm{M}+\mathrm{H}]^{+}: 370$, Found 370; Yield: $80 \%$.

1,3,8-trihydroxy-6-methyl-2-(piperazin-1-ylmethyl) anthracene-9, 10-dione (19): IR (KBr) 3463, 3020, 2929, 2356, 1720, 1649, 1458, 1380, 1284, 1217, 1123, 765, $670 \mathrm{~cm}^{-1}$; ${ }^{1} \mathrm{H}$ NMR $\left(\mathrm{CDCl}_{3}\right.$, $300 \mathrm{MHz}) \delta 12.78(\mathrm{~s}, \mathrm{OH}), 12.05(\mathrm{~s}, \mathrm{OH}), 7.66(\mathrm{~s}, 1 \mathrm{H}), 7.02(\mathrm{~s}, 1 \mathrm{H}), 6.86$ $(\mathrm{s}, 1 \mathrm{H}), 3.97(\mathrm{~s}, 2 \mathrm{H}), 3.03(\mathrm{t}, 4 \mathrm{H}), 2.52(\mathrm{t}, 2 \mathrm{H}), 2.48(\mathrm{t}, 2 \mathrm{H}), 2.32(\mathrm{~s}, 3 \mathrm{H})$, 2.10(s, $1 \mathrm{H})$; ESI - MS: Cacld for $\mathrm{C}_{20} \mathrm{H}_{20} \mathrm{~N}_{2} \mathrm{O}_{5}[\mathrm{M}+\mathrm{H}]^{+}: 369$, Found 369; Yield: $75 \%$.

1, 3, 8-trihydroxy-6-methyl-2-((4-methylpiperazin-1-yl) methyl) anthracene-9, 10-dione (20): IR (KBr) 3484, 3021, 2403, 1678, 1526, $1427,1216,929,762,672 \mathrm{~cm}^{-1} ;{ }^{1} \mathrm{H}$ NMR (CDCl \& DMSO-d ${ }^{6}, 300 \mathrm{MHz}$ ) $\delta 7.84(\mathrm{~s}, 1 \mathrm{H}), 7.69(\mathrm{~s}, 1 \mathrm{H}), 7.20(\mathrm{~s}, 1 \mathrm{H}), 4.11(\mathrm{~s}, 2 \mathrm{H}), 2.92(\mathrm{t}, 4 \mathrm{H})$, $2.76(\mathrm{t}, 4 \mathrm{H}), 2.61(\mathrm{~s}, 3 \mathrm{H}), 2.49(\mathrm{~s}, 3 \mathrm{H})) ;{ }^{13} \mathrm{C} \mathrm{NMR}(\mathrm{CDCl} 3$ \& DMSO, $75 \mathrm{MHz})$ 190.62, 189.40, 168.45, 163.03, 162.67, 148.47, 134.58, 133.71, $124.73,121.31,113.25,110.66,108.59,54.88,53.96,52.92,46.22,22.58$; ESI - MS: Cacld for $\mathrm{C}_{21} \mathrm{H}_{22} \mathrm{~N}_{2} \mathrm{O}_{5}[\mathrm{M}+\mathrm{H}]^{+}: 383$, Found 383; Yield: 78\%.

2-((4-(4-fluorophenyl) piperazin-1-yl) methyl)-1, 3, 8-trihydroxy-6-methylanthracene-9, 10-dione (21): IR (KBr) 3557, 2931, 2837, 2355, 1650, 1510, 1455, 1367, 1222, 1018, 926, 766, $669 \mathrm{~cm}$ ${ }^{1} ;{ }^{1} \mathrm{H}$ NMR $\left(\mathrm{CDCl}_{3}, 300 \mathrm{MHz},\right) \delta 12.66(\mathrm{~s}, \mathrm{OH}), 12.05(\mathrm{~s}, \mathrm{OH}), 7.52(\mathrm{~s}$, $1 \mathrm{H}), 7.15(\mathrm{~s}, 1 \mathrm{H}), 6.98(\mathrm{~s}, 1 \mathrm{H}), 6.89(\mathrm{~m}, 4 \mathrm{H}), 3.93(\mathrm{~s}, 2 \mathrm{H}), 3.13(\mathrm{t}, 4 \mathrm{H})$, $2.77(\mathrm{t}, 4 \mathrm{H}), 2.36(\mathrm{~s}, 3 \mathrm{H})$; ESI - MS: Cacld for $\mathrm{C}_{26} \mathrm{H}_{23} \mathrm{FN}_{2} \mathrm{O}_{5}[\mathrm{M}+\mathrm{H}]^{+}$: 463, Found 463; Yield: 80\%.

General method for esterification and acid formation (Method C): A stirred solution of compound 1 (100mg, 0.00037 moles) in pyridine $(2 \mathrm{~mL})$ and acetic anhydride $(0.00185$ moles $)$ at $60-70^{\circ} \mathrm{C}$ for $4 \mathrm{hr}$. The reaction mixture was put into cold water for crystallization, then filtered and dried. To the resultant crude acetate was gradually added $10 \mathrm{ml}$ of acetic anhydride and glacial acetic acid mixture (1:1) and $\mathrm{CrO}_{3}$ at $45^{\circ} \mathrm{C}$ and stirred for $10 \mathrm{hr}$ at $70^{\circ} \mathrm{C}$. Acetic anhydride and glacial acetic acid mixture was removed by vacuum. It was then extracted with ethyl acetate $(3 \times 25 \mathrm{~mL})$, the organic layer was washed with water, dried over anhydrous $\mathrm{Na}_{2} \mathrm{SO}_{4}$ and evaporated under reduced pressure. Then the crude product was chromatographed on silica gel to afford the desired compound.

(E)-4,5-dihydroxy-7-methyl-9,10-dioxo-9, 10-dihydroanthracen-2-yl 2-methylbut-2-enoate (22): IR (KBr) 3408, 3023, 2929, 2370, $1731,1639,1464,1373,1217,1119,926,764,672 \mathrm{~cm}^{-1} ;{ }^{1} \mathrm{H}$ NMR $\left(\mathrm{CDCl}_{3}\right.$ $300 \mathrm{MHz}) 12.57(\mathrm{~s}, \mathrm{OH}), 12.23(\mathrm{~s}, \mathrm{OH}), 8.02(\mathrm{~s}, 1 \mathrm{H}), 8.01(\mathrm{~s}, 1 \mathrm{H}), 7.63$ $(\mathrm{s}, 1 \mathrm{H}), 7.33-7.19(\mathrm{q}, 1 \mathrm{H}), 7.11(\mathrm{~s}, 1 \mathrm{H}), 2.46(\mathrm{~s}, 3 \mathrm{H}), 2.05(\mathrm{~s}, 3 \mathrm{H}), 1.98(\mathrm{~d}$, $3 \mathrm{H})$; ESI - MS: Cacld for $\mathrm{C}_{20} \mathrm{H}_{16} \mathrm{O}_{6}[\mathrm{M}+\mathrm{H}]^{+}: 352$, Found 352; Yield: $65 \%$. 6-methyl-9, 10-dioxo-9,10-dihydroanthracene-1,3,8-triyl triacetate (23): $\mathrm{IR}(\mathrm{KBr}) 3407,2927,1761,1668,1605,1457,1324,1206$, 1029, 908, 766, $674 \mathrm{~cm}^{-1} ;{ }^{1} \mathrm{H}$ NMR $\left(\mathrm{CDCl}_{3} 300 \mathrm{MHz}\right) 8.01(\mathrm{~s}, 1 \mathrm{H}), 7.95$ (s, 1H), $7.23(\mathrm{~s}, 1 \mathrm{H}), 7.22(\mathrm{~s}, 1 \mathrm{H}), 2.50(\mathrm{~s}, 3 \mathrm{H}), 2.43(\mathrm{~s}, 6 \mathrm{H}), 2.35(\mathrm{~s}, 3 \mathrm{H})$; ESI - MS: Cacld for $\mathrm{C}_{21} \mathrm{H}_{16} \mathrm{O}_{8}[\mathrm{M}+\mathrm{H}]^{+}:$397, Found 397; Yield: $98 \%$.

4,5,7-triacetoxy-9,10-dioxo-9,10-dihydroanthracene-2carboxylic acid (24): IR (KBr) 3416, 3024, 2927, 2367, 1770, 1648, 1460, 1372, 1215, 1029, 923, 761, $672 \mathrm{~cm}^{-1} ;{ }^{1} \mathrm{H}$ NMR (DMSO-d $\left.\mathrm{d}^{6}, 300 \mathrm{MHz}\right)$ 8.12 (s, 1H), 7.95 (s, 1H), 7.93 (s, 1H), 7.63 (s, 1H), 2.39 (s, 9H); ${ }^{13} \mathrm{C}$ NMR (DMSO-d $\mathrm{d}^{6}, 75 \mathrm{MHz}$ ) 180.14, 179.37, 168.40, 166.29, 165.02, $154.69,150.99,149.81,136.56,135.26,134.43,132.51,124.98,123.96$, $123.08,121.93,120.39,20.88$; ESI - MS: Cacld for $\mathrm{C}_{21} \mathrm{H}_{14} \mathrm{O}_{10}[\mathrm{M}+\mathrm{H}]^{+}$: 425, Found 425; Yield: $80 \%$.

\section{Biological Study}

\section{Materials}

RPMI-1640 medium, sodium bicarbonate, glucose, hypoxanthine, resazurin, chloroquine diphosphate and MEM medium were purchased from Sigma (St. Louis, MO, USA). Albumax II was procured from Gibco BRL (Grand Island, NY, USA). Giemsa stain was purchased from Merck (USA). SYBR Green 1 nucleic acid gel stain was purchased from Invitrogen molecular probe (Carlsbad, USA).

\section{In-vitro cultivation of $P$. falciparum}

The chloroquine sensitive ( $P f 3 \mathrm{D} 7)$ and chloroquine resistant $(P f \mathrm{~K} 1)$ strains of $P$. falciparum were grown in continuous culture according to Trager and Jensen [12]. Parasites were cultured in RPMI-1640 (HEPES modified) medium (Sigma) supplemented with 0.5\% AlbuMaxII, 0.2\% glucose, $0.2 \% \mathrm{NaHCO}_{3}$ and $15 \mu \mathrm{M}$ hypoxanthine. Parasite growth rate and stage was determined by the examination of Giemsa's stained thin smears of the RBCs.

\section{Antimalarial activity of compounds}

In-vitro assessment of antimalarial activity of compounds towards $P$. falciparum was performed by the determination of fifty percent inhibitory concentration ( $\mathrm{IC}_{50}$ ) according to the method of Johnson [13] with some modifications. Compounds were tested in range between 20 $\mu \mathrm{M}$ to $0.31 \mu \mathrm{M}$. To determine $\mathrm{IC}_{50}$ initially $20 \mu \mathrm{M}$ concentration were used and two fold serially diluted till $0.31 \mu \mathrm{M}$ and chloroquine were prepared in 96 well plates and then $50 \mu \mathrm{l}$ asynchronous culture of infected erythrocytes with 1-1.5\% parasitaemia and 2-3\% haematocrit was added to each well $(100 \mu \mathrm{l}$-final volume). Eight wells were treated as positive control (without drug) and 4 wells as negative controls (without parasite and drug). These plates were incubated in $\mathrm{CO}_{2}$ incubator maintained at $37^{\circ} \mathrm{C}$ for $72 \mathrm{~h}$. After $72 \mathrm{~h}, 100 \mu \mathrm{l}$ lytic buffer containing SYBR Green 1X final concentration was added to each well and incubated for $1-2 \mathrm{~h}$ at room temperature in dark. Plates were read under fluorescence reader (Synergy HT BioTek) at Ex. 485nm, Em. $535 \mathrm{~nm}$. $\mathrm{IC}_{50}$ was determined on the basis of DNA content of the parasite by using MS-Excel template.

\section{Cytotoxicity assay}

Cytotoxic level of active compounds would be assessed according to protocol defined by O'Brien [14], with some modifications. The monkey kidney cell line will be maintained in vitro in MEM medium supplied with $15 \%$ Fetal Bovine Serum (FBS) and $5 \% \mathrm{CO}_{2}$ at $37^{\circ} \mathrm{C}$. An appropriate serial drug dilution was prepared in culture plates and the cells were exposed to these concentrations of particular compounds for two days, $10 \%$ of cell viability marker resazurin was added and read under fluorescent reader at excitation wavelength $530 \pm 25 \mathrm{~nm}$ and emission at $590 \pm 25 \mathrm{~nm}$ for 
calculation of the median cytotoxic concentration $\left(\mathrm{CC}_{50}\right)$. The selective index (SI) would be calculated by using the formula-

Selective index $=\frac{\text { Median cytotoxic concentration }\left(\mathrm{CC}_{50}\right)}{\text { Median inhibitory concentration }\left(\mathrm{IC}_{50}\right)}$

\section{Statistical analysis}

Fifty percent inhibitory concentration $\left(\mathrm{IC}_{50}\right)$ of tested compounds were obtained by transferring the data into a graphic program (e.g. Excel) and expressed as percentage of the untreated controls and then evaluated by Logit regression analysis using pre-programmed Excel spreadsheet obtained from MMV group at Swiss Tropical Institute, Basel, Switzerland [15].

\section{Results and Discussion}

\section{Chemistry}

In order to prepare O-alkylated derivatives (2-6), (7-15) hydroxyl group at meta position of emodin (1) was alkylated with various amine side chains (Figure 1) and long chain alkyl halides (Figure 1) under basic conditions using $\mathrm{K}_{2} \mathrm{CO}_{3}$ and DMF as a solvent.

To study the substituent effect on anthraquinone nucleus C-alkylated derivates 16-21 were prepared using Mannich reaction protocol [16-20] (Figure 2).

"Since the emodin (1) contain free hydroxyl group we planned to" prepare few esters to study their antimalarial activity. To prepare ester derivative $\mathbf{2 2}$ and $\mathbf{2 3}$, emodin (1) was reacted with tiglic acid in presence of triethylamine at $0^{\circ} \mathrm{C}$ and acetic anhydride in presence of pyridine respectively. To transform the methyl group in to acid functionality,

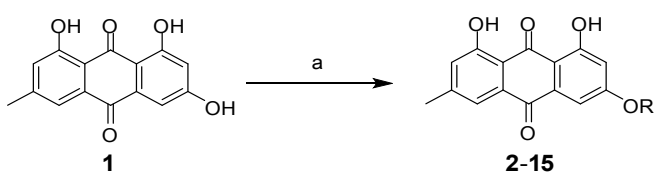

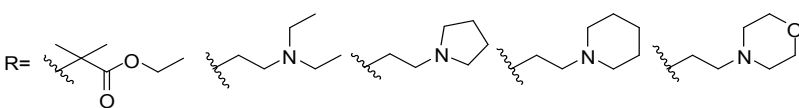

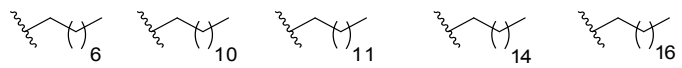

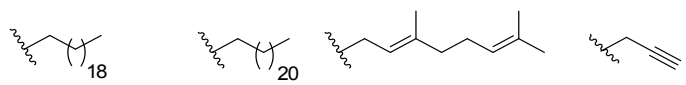

Reagents and conditions: a) $\mathrm{RX}, \mathrm{K}_{2} \mathrm{CO}_{3}, \mathrm{DMF}, 60^{\circ} \mathrm{C}$, 4hrs.

Figure 1: Preparation of O- alkylated derivatives 2-15 of emodin (1).

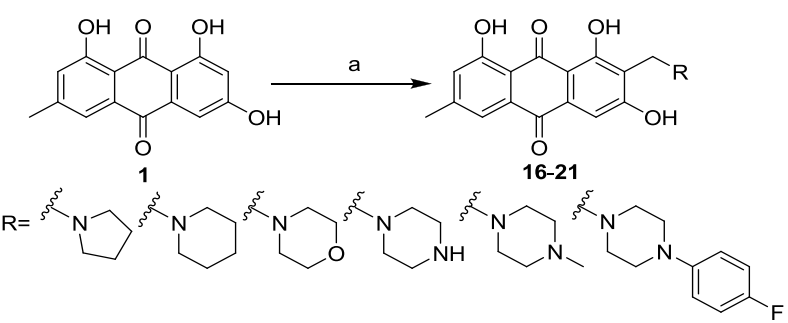

Reagents and conditions: a) Formalin, Amine, Gla AcOH, $0^{\circ} \mathrm{C}-\mathrm{rt}, 4 \mathrm{hrs}, 75-80 \%$.

\footnotetext{
Figure 2: Preparation of C-alkylated derivatives 16-21 of emodin (1).
}

emodin triacetate (23) was oxidized with chromium trioxide in presence of glacial acetic acid and acetic anhydride to provide acid (24).

\section{Biological evaluation}

In vitro antimalarial activity: All the compounds were screened

\begin{tabular}{|c|c|c|c|c|}
\hline \multirow{3}{*}{ Comp. No } & \multirow{3}{*}{ Chemical Structure } & \multicolumn{2}{|c|}{$\mathrm{IC}_{50}$} & \multirow{2}{*}{$\begin{array}{c}\mathrm{CC}_{50} \\
\text { VERO } \\
\text { cell line }\end{array}$} \\
\hline & & $\begin{array}{l}\text { Pf3D7 } \\
\text { (CQS) }\end{array}$ & $\begin{array}{c}\text { PfK1 } \\
\text { (CQR) }\end{array}$ & \\
\hline & & $\mu \mathrm{M}$ & $\mu \mathrm{M}$ & $\mu \mathrm{M}$ \\
\hline 1 & & 16.2 & 37 & nd \\
\hline 2 & & 16.2 & $>52$ & nd \\
\hline 3 & & 22.4 & 48.8 & nd \\
\hline 4 & & 12.6 & 30.6 & nd \\
\hline 5 & & 5.7 & 12.1 & 69.8 \\
\hline 6 & & 10.6 & 18.41 & nd \\
\hline 7 & & 36.5 & $>52.35$ & nd \\
\hline 8 & & $>45.6$ & $>45.6$ & nd \\
\hline 9 & & $>44.24$ & $>44.24$ & nd \\
\hline 10 & & $>40.4$ & $>40.4$ & nd \\
\hline 11 & & $>38.31$ & $>38.31$ & nd \\
\hline 12 & & 2.1 & $>36.36$ & 909 \\
\hline 13 & & $>34.6$ & $>34.6$ & nd \\
\hline 14 & & $>49.26$ & $>49.26$ & nd \\
\hline
\end{tabular}




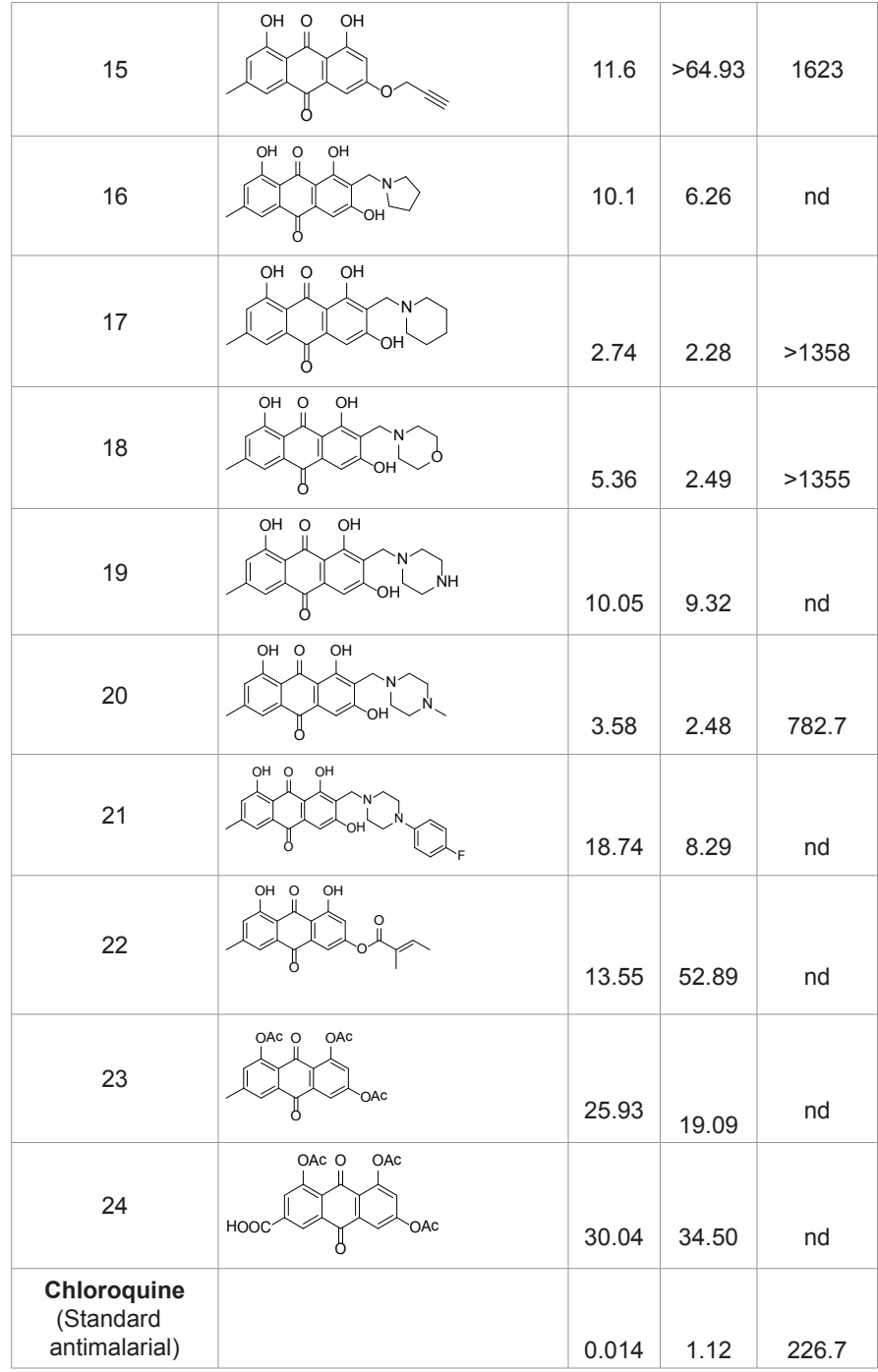

nd= not determined

Table 1: Chemical structures and in vitro antimalarial activity $\left(\mathrm{IC}_{50}\right.$ in $\left.\mu \mathrm{M}\right)$ of emodin (1) and its derivatives (2-24)

against chloroquine sensitive (Pf3D7) and chloroquine resistant (PfK1) strain of $P$. falciparum. The parent compound, emodin (1) has an $\mathrm{IC}_{50}$ of 16.2 and $37 \mu \mathrm{M}$ against $P f 3 \mathrm{D} 7$ and $P f K 1$ strain respectively (Table 1 ). Except 2, 3, 7-11, 13, 14 \& 24 all other derivatives exhibited improved in-vitro antimalarial activity against $P f 3 \mathrm{D} 7$ and $P f \mathrm{~K} 1$ strains with better therapeutic index (Table 1). Derivatives $4\left(\mathrm{IC}_{50}=12.6 \mu \mathrm{M}\right), \mathbf{6}\left(\mathrm{IC}_{50}=10.6\right.$ $\mu \mathrm{M}), 15\left(\mathrm{IC}_{50}=11.6 \mu \mathrm{M}\right), 16\left(\mathrm{IC}_{50}=10.1 \mu \mathrm{M}\right), 19\left(\mathrm{IC}_{50}=10.05 \mu \mathrm{M}\right), 22$ $\left(\mathrm{IC}_{50}=13.55 \mu \mathrm{M}\right)$ shows moderate activity against $P f 3 \mathrm{D} 7$. Derivatives $5\left(\mathrm{IC}_{50}=12.1 \mu \mathrm{M}\right), 23\left(\mathrm{IC}_{50}=19.09 \mu \mathrm{M}\right)$ shows moderate activity against PfK1. Derivatives $5\left(\mathrm{IC}_{50}=5.7 \mu \mathrm{M}\right), \mathbf{1 2}\left(\mathrm{IC}_{50}=2.1 \mu \mathrm{M}\right)$ have $\mathrm{IC}_{50}$ below $10 \mu \mathrm{M}$ against Pf3D7. Derivatives $16\left(\mathrm{IC}_{50}=6.26 \mu \mathrm{M}\right), 19\left(\mathrm{IC}_{50}=9.32\right.$ $\mu \mathrm{M}), 21\left(\mathrm{IC}_{50}=8.29 \mu \mathrm{M}\right)$ have $\mathrm{IC}_{50}$ below $10 \mu \mathrm{M}$ against $P f \mathrm{~K} 1$. Where as derivatives $17\left(\mathrm{IC}_{50}=2.74 \mu \mathrm{M}, 2.28 \mu \mathrm{M}\right), 18\left(\mathrm{IC}_{50}=5.36 \mu \mathrm{M}, 2.49\right.$ $\mu \mathrm{M}), 20\left(\mathrm{IC}_{50}=3.58 \mu \mathrm{M}, 2.48 \mu \mathrm{M}\right)$ also have $\mathrm{IC}_{50}$ below $10 \mu \mathrm{M}$ against $P f 3 \mathrm{D} 7$ and $P f \mathrm{~K} 1$ strain. Our structure activity relationships indicated that C-alkylation of emodin (1) improves the activity in both strains, where as O-alkylation only improves the activity in Pf3D7 strain. It is noteworthy to mention here that Mannich base derivatives 17,18 and 20 exhibited comparable in-vitro antimalarial activity with the marketed drug chloroquine $\left(\mathrm{IC}_{50}=1.12\right)$ with good therapeutic index against CQ resistant strain (Table 2, Figure 3 and 4).

\begin{tabular}{|c|c|c|c|c|}
\hline Compound No. & $\begin{array}{c}\text { IC }_{50} \\
(\mathbf{P f 3 D})(\boldsymbol{\mu M})\end{array}$ & $\begin{array}{c}\mathbf{I C}_{\mathbf{5 0}^{5}} \\
(\mathbf{P f k} \mathbf{)}(\boldsymbol{\mu M})\end{array}$ & $\begin{array}{c}\text { SI (Pf3D7) } \\
(\boldsymbol{\mu} \mathbf{M})\end{array}$ & $\begin{array}{c}\text { SI (Pfk1) } \\
(\boldsymbol{\mu M})\end{array}$ \\
\hline 17 & 2.74 & 2.28 & 495.6 & 595.6 \\
\hline 18 & 5.36 & 2.49 & $>252$ & $>544$ \\
\hline 20 & 3.58 & 2.48 & 218.6 & 315 \\
\hline Chloroquine & 0.014 & 1.12 & 16192.8 & 201.7 \\
\hline
\end{tabular}

Table 2: Antimalarial profile and safety index of active compounds against CQS (Pf3D7) and CQR (PfK1) strains of P.falciparum.

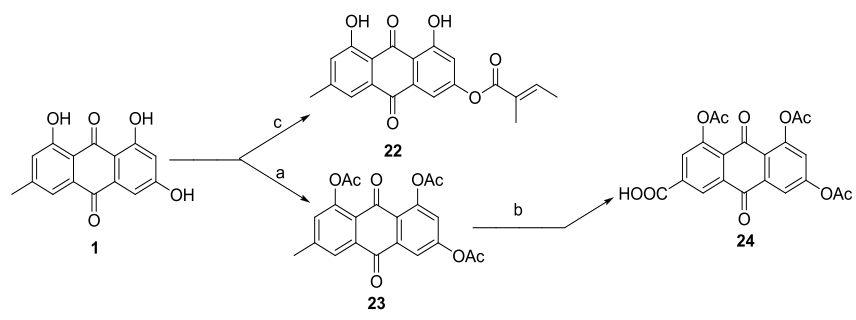

Reagents and conditions: a) $\mathrm{Ac}_{2} \mathrm{O}$, Pyridine, $60-70^{\circ} \mathrm{C}$
10hrs, $80 \%$ c) RCOCl, $\mathrm{Et}, \mathrm{N}, \mathrm{DCM}, \mathrm{O}^{\circ} \mathrm{C}$, $2 \mathrm{hrs}, 65 \%$

Figure 3: Preparation of ester derivatives 22, 23 and acid (24) from emodin (1).

\section{A Selective Index of active compounds for pf3D7}

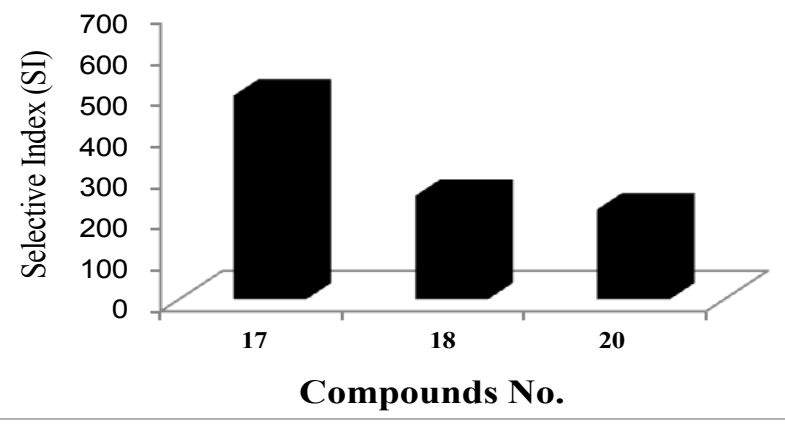

B Selective Index of active compounds for $p f k 1$

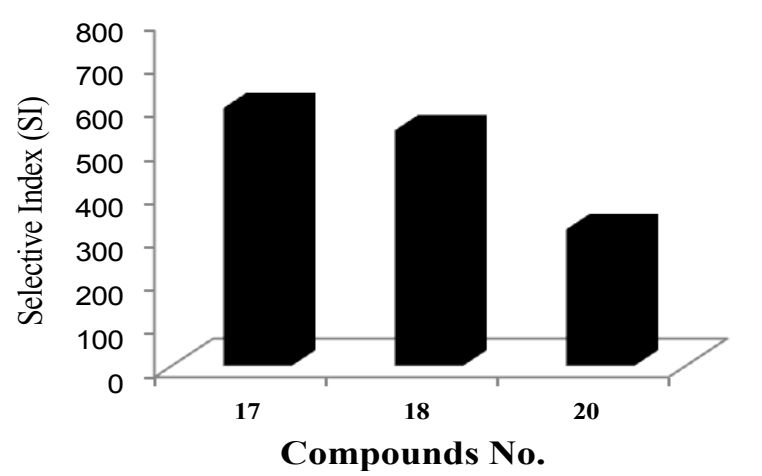

Figure 4: (A) Selective Index of active compounds for Pf3D7 (B). Selective Index of active compounds for PfK1. 


\section{Cytotoxicity assay and selective index (SI)}

In order to characterize the Pf3D7 and PfK1 strain basis of antimalarial effects of selected derivatives $5,12,15,17,18$ and 20 we investigated the cytotoxic level of these active compounds. Derivatives $\mathbf{5}\left(\mathrm{CC}_{50}=69.8 \mu \mathrm{M}\right), \mathbf{1 2}\left(\mathrm{CC}_{50}=909 \mu \mathrm{M}\right), \mathbf{1 5}\left(\mathrm{CC}_{50}=1623 \mu \mathrm{M}\right), \mathbf{1 7}\left(\mathrm{CC}_{50}=\right.$ $>1358 \mu \mathrm{M}), \mathbf{1 8}\left(\mathrm{CC}_{50}=>1355 \mu \mathrm{M}\right), 20\left(\mathrm{CC}_{50}=782.7 \mu \mathrm{M}\right)$ against $\mathrm{VERO}$ cell line. These compounds did not show any significant cytotoxicity against vero cell line (Table 4 ).

\section{Conclusions}

In conclusion, we have isolated larger quantities of emodin (1) from the roots of Rheum emodi and a library of novel emodin O-alkylated derivatives 2-6, 7-15, C-alkylated Mannich derivatives 16-21, acyl derivatives 22-23, and acid derivative 24 (Figure 3 ) from 1 were synthesized and evaluated their in-vitro antimalarial activity against chloroquine sensitive strain and chloroquine resistant strain. Among these 24 derivatives, C-alkyl Mannich bases 17, 18 and 20 showed potent antimalarial activity against chloroquine resistant strain PfK1with an $\mathrm{IC}_{50}$ of $2.28,2.49,2.48 \mu \mathrm{M}$ respectively, which is comparable to marketed drug chloroquine ( $\mathrm{IC}_{50}$ of 1.12). Further work is in progress in our laboratory to prepare more Mannich bases of emodin to develop a potent lead for inhibition of chloroquine resistant malaria.

\section{Acknowledgment}

Authors are thankful to Director, CSIR-CDRI for constant encouragement for the program on natural products of biological importance. We also thank the ICMR, New Delhi for providing research grant and fellowship. SAIF, CDRI for spectral data. Financial support from SPlenDID is acknowledged.

\section{References}

1. Zaprometnov MN (1993) Phenolic Compounds. Distribution, Metabolism, and Functions in Plants. USSR Academy of sciences (Nauka), Moscow.

2. Gorelik MV (1983) Chemistry of Anthraquinone and Its Derivatives. USSR Academy of sciences, Moscow.

3. Fain VYa (1999) 9, 10-Anthraquinones and their Use. USSR Academy of sciences, Moscow.

4. Alves DS, Pérez-Fons L, Estepa A, Micol V (2004) Membrane-related effects underlying the biological activity of the anthraquinones emodin and barbaloin. Biochem Pharmacol 68: 549-561.
5. Liang JW, Hsiu SL, Huang HC, Lee-Chao PD (1993) HPLC analysis of emodin in serum, herbs and Chinese herbal prescriptions. Journal of Food and Drug Analysis 1: 251-257.

6. Yang F, Zhang T, Tian G, Cao H, Liu Q, Ito Y (1999) Preparative isolation and purification of hydroxyl anthraquinones from Rheum officinale Baill by highspeed counter-current chromatography using $\mathrm{pH}$-modulated stepwise elution. Journal of Chromatography A 858: 103-107.

7. Narender T, Sukanya P, Komal Sharma, Surendar Reddy Bathula (2013) Preparation of novel antiproliferative emodin derivatives and studies on their cellcycle arrest, caspase dependent apoptosis and DNA binding interaction. Phytomedicine 20: 890-896.

8. Kuo YC, Meng HC, Tsai WJ (2001) Regulation of cell proliferation, inflammatory cytokine production and calcium mobilization in primary human T lymphocytes by emodin from Polygonum hypoleucum Ohwi. Inflamm Res 50: 73-82.

9. Huang HC, Chu SH, Chao PD (1991) Vasorelaxants from Chinese herbs emodin and scoparone, possess immunosuppressive properties. Eur J Pharmacol 198: 211-213.

10. Zhou XM, Chen QH (1998) Biochemical study of Chinese rhubarb. XXII. Inhibitory effect of anthraquinone derivatives on $\mathrm{Na}+-\mathrm{K}+-\mathrm{ATP}$ ase of the rabbit renal medulla and their diuretic action. Acta Pharmaceutica Sinica 23: 17-20.

11. Koyama M, Kelly TR, Watanabe KA (1988) Novel type of potential anticancer agents derived from chrysophanol and emodin. Some structure-activity relationship studies. J Med Chem 31: 283-284.

12. Trager W, Jensen JB (1976) Human malaria parasites in continuous culture. Science 20: 673-675.

13. Johnson J D, Richard AD, Lucia G, Miriam L, Norma ER, Norman CW (2007) Assessment and Continued Validation of the Malaria SYBR Green I-Based Fluorescence Assay for Use in Malaria Drug Screening. Antimicrobial Agents Chemother 51: 1926

14. O'Brien J, Wilson I, Orton T, Pognan F (2000) Investigation of the Alamar Blue (resazurin) fluorescent dye for the assessment of mammalian cell cytotoxicity. Eur J Biochem 267: 5421-5426.

15. Pandey SK, Dwivedi H, Singh S, Siddiqui WA, Tripathi R (2013) Antimalaria interaction of quinine and quinidine with clarithromycin. Parasitology 140: 406-413.

16. Kemertelidze EP, Gotsiridze AV (1971) Khimiya Prirodnykh Soedinenii 519.

17. Kemertelidze EP, Gotsiridze AV (1971) Soobshcheniya Akademii Nauk Gruzii. SSR 61: 93

18. Blicke FF (1942) Mannich reaction. Organic Reaction 1: 303.

19. Brewster JH, Eliel EL (1953) Carbon-carbon alkylation of amines and ammonium salts. Organic Reactions 8.

20. Kintsurashvili LA, Sikharulidze MI, Buyanov VN, Turabelidze DG (1999) Synthesis of amino derivatives of 1, 6 8-trihydroxy-3-methyl-9, 10-anthraquinone.Chemistry of Natural Compounds 35: 619-620. 